\title{
THE IMPACT OF ANTINUCLEAR ANTIBODIES INDUCED BY ANTITUMOR NECROSIS FACTOR ALPHA (ANTI-TNF- $\alpha$ ) AGENTS ON THE LONG-TERM TREATMENT OUTCOMES IN RHEUMATOID ARTHRITIS PATIENTS
}

\author{
Daniela Oliveira ${ }^{1, \star}$, Ana Martins ${ }^{1}$, Frederico Martins ${ }^{2}$, Maria Rato ${ }^{1}$, Filipe Pinheiro ${ }^{1}$, Diogo Fonseca ${ }^{3}$, Salomé Garcia ${ }^{1}$, Bruno \\ Fernandes ${ }^{1}$, Carlos Vaz ${ }^{1}$, Miguel Bernardes ${ }^{1}$, Lúcia Costa ${ }^{1}$ \\ 1.Centro Hospitalar Universitário de São João, Porto (Douro Litoral), Portugal; 2.Centro Hospitalar Universitário do Algarve, Faro (Algarve), \\ Portugal; 3.Centro Hospitalar de Vila Nova de Gaia/Espinho, Vila Nova de Gaia (Douro Litoral), Portugal.
}

*Corresponding author: danielasoff@gmail.com

\section{BACKGROUND}

The seroconversion of antinuclear antibodies (ANA) induced by anti-TNF- $\alpha$ therapy remains a matter of concern in rheumatoid arthritis (RA). However, evidence is still scarce regarding the impact of these autoantibodies on the clinical response to treatment. This study aimed to explore the impact of ANA induced by anti-TNF- $\alpha$ therapy on the outcomes of treatment in patients with RA over two years of follow-up.

\section{METHODS}

An observational retrospective cohort study was conducted with two years of follow-up. Patients diagnosed with RA, according to the American College of Rheumatology (ACR) criteria, and registered on the Rheumatic Diseases Portuguese Register (Reuma. pt) who started their first anti-TNF- $\alpha$ agent as first biologic between 2003 and 2018 were included. Patients with positive ANA (titer $\geq 100$ ) and/or positive anti-dsDNA antibodies and/or with a diagnosis of SLE at their first visit were excluded. Demographic, clinical and laboratory data were obtained by consulting Reuma.pt. disease activity score for 28 joints (DAS28), DAS28 delta, health assessment questionnaire (HAQ), HAQ delta were assessed at baseline, 6, 12, 18 and 24 months. Clinical response was evaluated by EULAR criteria and three response categories were defined: good, mild and no response. The rate of switch of biological treatment was assessed over 24 months. To examine the differences between groups with and without ANA seroconversion independent samples t test, Mann-Whitney U-tests and Chi-square tests were used. Logistic regression models were used to assess the effects of ANA seroconversion on clinical response to treatment over time.

\section{RESULTS}

A total of 185 patients (mean age of $49.3 \pm 10.9$ years old; $85.4 \%$ female) with a median follow-up of 7 (4-14) years were included. We found an ANA seroconversion rate (titer $\geq 100)$ of $77.3 \%(n=143)$ with median time of $36(15-72.3)$ months. There were no differences among groups regarding age, gender, disease duration, seropositivity or not and have an erosive disease or not. The DAS28 delta was significantly different $(p=0.035)$ between group with positive ANA $(2.01 \pm 1.29)$ and negative ANA (1.15 \pm 1.51$)$ at six months. The DAS28 was significantly different $(p=0.014)$ between group with positive ANA $(5.06 \pm 3.39)$ and negative ANA $(3.99 \pm 1.43)$ at 12 months. No statistically significant differences were found in other variables. Switch rate was significantly different between patients with ANA seroconversion (median 1 [0-1]) versus absence of seroconversion (median 0 [0-1]), $p=0.025$. In the regression model ANA seroconversion did not predict switch rate and EULAR response over time.

\section{CONCLUSION}

This study showed that the majority of patients with RA treated with an anti-TNF-a agent developed ANA and that their presence may be associated with worse clinical results over time. In fact, previous research suggested that a decrease in anti-TNF- $\alpha$ drug concentration due to the production of autoantibodies may lead to worse outcomes of treatment. Moreover, our data demonstrated that patients with ANA seroconversion had a higher switch rate. Despite these results, there are no differences in the EULAR response between the two groups and ANA seroconversion did not predict this response. Therefore, ANA induced by anti-TNF- $\alpha$ agents should be monitored in patients with RA and its impact on treatment must be considered. Further research is needed to explore these results.

\section{KEYWORDS}

Rheumatoid arthritis, Antinuclear antibodies, Antitumor necrosis factor alpha agent, Anti-TNF-a agents, Outcomes. 